\title{
Microwave Enhanced Cotunneling in SET Transistors
}

\author{
Manscher, Martin; Savolainen, M.; Mygind, Jesper
}

Published in:

I E E E Transactions on Applied Superconductivity

Link to article, DOI:

10.1109/TASC.2003.814167

Publication date:

2003

Document Version

Publisher's PDF, also known as Version of record

Link back to DTU Orbit

Citation (APA):

Manscher, M., Savolainen, M., \& Mygind, J. (2003). Microwave Enhanced Cotunneling in SET Transistors. I E E E Transactions on Applied Superconductivity, 13(2), 1107-1110. https://doi.org/10.1109/TASC.2003.814167

\section{General rights}

Copyright and moral rights for the publications made accessible in the public portal are retained by the authors and/or other copyright owners and it is a condition of accessing publications that users recognise and abide by the legal requirements associated with these rights.

- Users may download and print one copy of any publication from the public portal for the purpose of private study or research.

- You may not further distribute the material or use it for any profit-making activity or commercial gain

- You may freely distribute the URL identifying the publication in the public portal

If you believe that this document breaches copyright please contact us providing details, and we will remove access to the work immediately and investigate your claim. 


\title{
Microwave Enhanced Cotunneling in SET Transistors
}

\author{
M. H. Manscher, M. T. Savolainen, and J. Mygind
}

\begin{abstract}
Cotunneling in single electron tunneling (SET) devices is an error process which may severely limit their electronic and metrologic applications. Here is presented an experimental investigation of the theory for adiabatic enhancement of cotunneling by coherent microwaves. Cotunneling in SET transistors has been measured as function of temperature, gate voltage, frequency, and applied microwave power. At low temperatures and applied power levels, including also sequential tunneling, the results can be made consistent with theory using the unknown damping in the microwave line as the only free parameter.
\end{abstract}

\section{INTRODUCTION}

$\mathbf{S}$ INGLE electron tunneling (SET) devices have unique properties that have opened applications such as current and capacitance standards [1], [2], electrometers [3], and thermometry [4]. They also may be used within quantum logic and quantum computing. The first-order behavior is adequately described by the orthodox theory [6], [7]. The so-called cotunneling, a second-order phenomenon in which two electrons tunnel at the same time, constitutes an important error process in applications. The dependence of the cotunneling current on voltage and temperature has been studied theoretically [8], [9] as well as experimentally [10]-[12]. Furthermore, Covington et al. [13] have studied the frequency dependence for 4- and 6 -junction pumps. This paper investigates experimentally the theoretical prediction by Flensberg [14] that the cotunneling current should depend not only on temperature and voltage, but also on the amplitude and frequency of an applied oscillating field.

\section{THEORY}

The inelastic cotunneling current in a single SET transistor for low temperature $\left(k_{\mathrm{B}} T_{\mathrm{e}} \ll \Delta^{ \pm}\right)$and bias voltage $\left(e V_{\mathrm{DC}} \ll\right.$ $\Delta^{ \pm}$) was derived by Averin and Nazarov [8] as

$$
\begin{aligned}
I_{\mathrm{cot}}^{(3)}=\frac{R_{\mathrm{K}}}{24 \pi^{2} R_{1} R_{2}}\left(\frac{1}{\Delta^{+}}\right. & \left.+\frac{1}{\Delta^{-}}\right)^{2} \\
& \times\left[\left(2 \pi k_{\mathrm{B}} T_{\mathrm{e}}\right)^{2}+\left(e V_{\mathrm{DC}}\right)^{2}\right] V_{\mathrm{DC}} .
\end{aligned}
$$

Here, $R_{1,2}$ are the tunneling resistances of the left and right electrodes, respectively, $\Delta^{ \pm}=\left(e / C_{\Sigma}\right)\left(e / 2 \mp C_{\mathrm{G}} V_{\mathrm{G}}\right)(\bmod$ $\left.\left(e^{2} / C_{\Sigma}\right)\right)$ are the energies to add/remove one electron to/from

Manuscript received August 4, 2002. This work was supported in part by the Hartman Foundation, the Danish Natural Science Foundation, and EU contract IST-1999-10673. The work of M. T. Savolainen was also supported by a grant from Tekniikan Edistämissäätiö, TES.

M. H. Manscher and J. Mygind are with the Department of Physics, B309, Technical University of Denmark, DK-2800 Lyngby, Denmark (e-mail: manscher@fysik.dtu.dk; myg@fysik.dtu.dk).

M. T. Savolainen is with the Department of Physics, University of Jyväskylä, FIN-40351 Jyväskylä, Finland (e-mail: mtsavola@cc.jyu.fi).

Digital Object Identifier 10.1109/TASC.2003.814167 the island, $T_{\mathrm{e}}$ is the temperature of the electron system, and $R_{\mathrm{K}}=h / e^{2}$. In the maximum blockade state, $\Delta^{ \pm}$reduce to the charging energy $E_{\mathrm{C}}=e^{2} / 2 C_{\Sigma}$, where $C_{\Sigma}=C_{1}+C_{2}+C_{\mathrm{G}}$ is the total capacitance of the island to the surroundings (the ground capacitance is assumed negligible). Equation (1) has been verified experimentally in both metallic and 2-D semiconducting systems by several researchers [10]-[12].

Flensberg [14] has extended the analysis by Averin and Nazarov to the case where a harmonically varying signal $V_{\mathrm{AC}} \cos (2 \pi f t)$ is applied on top of the DC bias. His result for the cotunneling current, obtained by expansion to the third order in the energies, becomes in the adiabatic (low-frequency, low-temperature, low-amplitude) limit

$$
\begin{aligned}
I_{\mathrm{cot}}^{(3)}= & \frac{R_{\mathrm{K}}}{24 \pi^{2} R_{1} R_{2}}\left(\frac{1}{\Delta^{+}}+\frac{1}{\Delta^{-}}\right)^{2} \\
& \times\left[\left(2 \pi k_{\mathrm{B}} T_{\mathrm{e}}\right)^{2}+\left(e V_{\mathrm{DC}}\right)^{2}+\frac{3}{2}\left(e V_{\mathrm{AC}}\right)^{2}\right] V_{\mathrm{DC}} .
\end{aligned}
$$

In restating his equations we assume (as is the case for our experiments) that the alternating bias is applied to the left lead, while the right is kept at a constant potential.

Flensberg further derived also the next leading order correction to (2) by an expansion to fourth order in the energies:

$$
\begin{aligned}
I_{\mathrm{cot}}^{(4)}= & \frac{R_{\mathrm{K}}}{48 \pi^{2} R_{1} R_{2}}\left(\frac{1}{\Delta^{+}}+\frac{1}{\Delta^{-}}\right)^{2}\left(\frac{1}{\Delta^{-}}-\frac{1}{\Delta^{+}}\right) \\
& \times\left[\left(2 \pi k_{\mathrm{B}} T_{\mathrm{e}}\right)^{2}+\frac{3}{4}\left(e V_{\mathrm{AC}}\right)^{2}+(h f)^{2}\right]\left(e V_{\mathrm{AC}}\right)^{2} .
\end{aligned}
$$

The temperature, amplitude and frequency are assumed so low that an expansion in powers of these is appropriate. Note that in the case of maximum Coulomb blockade, $I_{\text {cot }}^{(4)}$ vanishes, since then $\Delta^{ \pm}=E_{\mathrm{C}}$ and thus the third multiplicative term becomes zero.

The aim of the present work is to experimentally verify or disprove (2). We do this by applying microwaves to the device and measuring the differential conductance by a lock-in technique at zero DC bias. This ensures that the current predicted by (3) does not contribute to the result.

The sequential tunneling and cotunneling conductances for a SET transistor in zero bias and maximum Coulomb blockade is calculated with the Master Equation approach in the orthodox theory, using the measured parameters for the sample (JYU NM3) with which we made the cotunneling experiments. In the temperature range of interest it shows that the two conductances have the same order of magnitude, which means that sequential tunneling should be taken into account when interpreting the results.

\section{EXPERIMENTAL SETUP}

The measurements were made in a KelvinOx ${ }^{\mathrm{TM}}$ dilution refrigerator (loaded base temperature $50 \mathrm{mK}$ ) set up in a shielded room, with the mechanical pumps outside the shielding. The 
temperature of the mixing chamber (MC) could be measured with calibrated germanium and ruthenium-oxide thermometers. A $1 \mathrm{~T}$ field from a superconducting magnet run in persistent mode suppresses the superconductivity of the aluminum films of the SET transistor.

The samples were DC current biased symmetrically using a balanced voltage supply in series with two $10 \mathrm{M} \Omega$ resistors. A low-noise high input impedance FET voltage amplifier was used to measure the voltage across the sample. To measure dynamic resistances, a $10 \mathrm{pA}, 2 \mathrm{~Hz}$ modulation current from the output of a lock-in amplifier (SR850) was applied on top of the DC bias. The modulation voltage response was fed back to the lock-in amplifier input. With the measured zero-bias conductances, the excitation corresponds in the worst case ( $2 \mu \mathrm{S}$ differential conductance) to $50 \mathrm{aW}$ heat input, and the voltage magnitude of the excitation is $5 \mu \mathrm{V}$, corresponding to about $0.03 V_{\mathrm{C}}$, where $V_{\mathrm{C}}$ is the blockade voltage. All electrical connections to the sample are miniature coaxial cables with high leakage resistance and low cross-talk. The high capacitance in the filters and coax cables forces us to use quite low modulation frequencies. In all experiments the a worst-case cut-off is $8 \mathrm{~Hz}$. Details of the electronics setup are given elsewhere [15].

A separate coaxial transmission line is used to apply microwaves to the device. The construction is described elsewhere [15]. The line is thermally anchored at $4.2 \mathrm{~K}, 1.2 \mathrm{~K}, 0.6 \mathrm{~K}$ and at the MC. It includes three inside/outside DC breaks and cooled attenuators at $4.2 \mathrm{~K}$ and at $\mathrm{MC}$ temperature. As a consequence of the distributed attenuation and possible resonances in the microwave transmission line and wiring to the SET device, the actual power applied to the sample is unknown, and all power values are measured at the output of the microwave synthesizer connected to the $300 \mathrm{~K}$ end of the line. As described below we use the SET transistor as a self-detector and the total attenuation of the line at each frequency will be a fitting parameter in the data.

Pressures and temperatures in the ${ }^{3} \mathrm{He}$ circulation system are continuously monitored by a computer, which also controls the biasing of the sample and reads the results measured by the voltage meters etc. through a GPIB bus. The GPIB bus is separated electrically from all measurement electronics by an optical link, which galvanically separates the computer from the measurement, and also enables the computer to be moved out of the shielded room if necessary.

\section{RESULTS}

\section{A. Device Characterization}

For the particular SET transistor reported on here, the DC $I-V_{\mathrm{DC}}$ curve yields the device parameters $R_{\Sigma}=R_{1}+R_{2}=$ $95.0 \mathrm{k} \Omega$ and $C_{\Sigma}=C_{1}+C_{2}=1030 \mathrm{aF}$. From the voltage versus gate voltage $\left(V_{\mathrm{DC}}-V_{\mathrm{G}}\right)$ curves we get $C_{\mathrm{G}}=0.94 \mathrm{aF}$. The set of $V_{\mathrm{DC}}-V_{\mathrm{G}}$ curves at different current bias also provided evidence that the device is nearly symmetric. The particular sample was chosen from a batch for its fairly high charging energy, which also means a higher tunneling resistance.

We want to first compare with the theory for zero current bias and maximum blockade. To ensure this we find the gate voltage giving maximum Coulomb blockade by sweeping across a peak

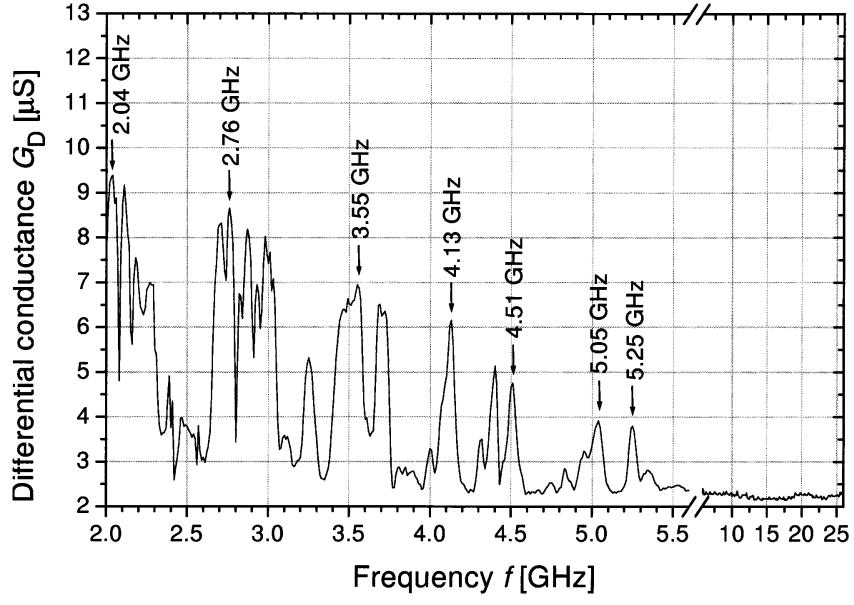

Fig. 1. Resonances in the high-frequency line. The SET is used as a self-detector by biasing in zero bias, maximum blockade and applying a fixed $-10 \mathrm{dBm}$ signal from the microwave synthesizer. The differential conductance is then recorded as a function of the frequency. Notice the break in the frequency scale.

in the $V_{\mathrm{DC}}-V_{\mathrm{G}}$ curve. Then, with this gate voltage fixed, the zero DC bias point was found by recording a $I-V_{\mathrm{DC}}$ curve around zero DC bias and fitting the dynamic resistance to find the peak. This procedure was carried out before each measurement of $G_{\mathrm{D}}$.

The microwave line exhibits natural resonances, at which the signal reaching the SET is higher. The resonances are determined using the SET device as a self-detector by measuring the dynamic resistance at zero DC bias and maximum blockade as function of frequency in small steps. The method is based on the assumption of a response both from the sequential tunneling and cotunneling contributions, as neither of these are linear around zero bias. The result is shown in Fig. 1, where clear peaks in the dynamic resistance are seen at various frequencies. The results shown in this paper are measured at the frequencies marked by arrows.

\section{B. Temperature of the Measurements}

The pertinent question relating to low temperature measurements is: What is the correct temperature? First, the sample may be far away from the MC chamber of the dilution refrigerator. This means that any heat delivered to the sample by Joule heating or radiation gives a higher temperature at the sample than at the MC. Second, there is the question of whether the electron system in the SET transistor is in thermal equilibrium with the phonon system [16]. This suggests that it is not correct to rely on the temperature measured by the thermometers fixed to the MC, even when these are well-calibrated.

To get an estimate of the real electron temperature, we use the results of the differential conductance measurements at maximum blockade and zero bias. Using both the orthodox theory and the cotunneling theory, the temperature has been optimized to make the differential conductance $G_{\mathrm{D}}$ fit. Here the junction parameters were fixed at the measured values determined in the previous section, leaving $T_{\mathrm{e}}$ as the only free parameter. The results of this temperature fitting is shown in Fig. 2 as function of the MC temperature $T_{\mathrm{ph}}$, which we assume (due to our careful thermal anchoring of the substrate and following [16]) to be equal to the phonon temperature, hence the subscript. The 


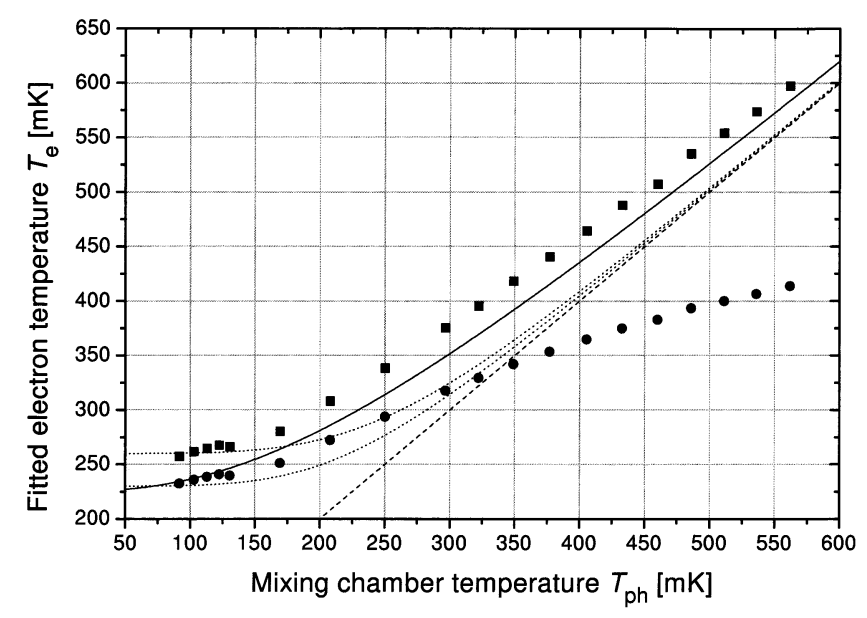

Fig. 2. Temperature estimates from the zero-bias conductance. The fit to the sequential tunneling only (squares) provides an upper bound, while the fit to sequential tunneling plus cotunneling (points) is a lower bound. The electron temperature should approach the mixing chamber temperature (dashed line) at high temperatures. Also shown are the forms $T_{\mathrm{e}}=\sqrt[3]{T_{0}^{3}+T_{\mathrm{ph}}^{3}}$ (solid line; $T_{0}=227 \mathrm{mK}$ ) and $T_{\mathrm{e}}=\sqrt[5]{T_{0}^{5}+T_{\mathrm{ph}}^{5}}$ (dotted lines; $T_{0}=260 \mathrm{mK}$ for the upper curve, $T_{0}=235 \mathrm{mK}$ for the lower curve).

temperature has been fit both to the sequential tunneling conductance alone (squares), which will give an upper bound on the temperature, and to the tunneling conductance with cotunneling included (circles), which will give a lower bound under the assumption that other effects can be neglected. We have implicitly assumed that we can use the theory for cotunneling by Averin and Nazarov at low temperatures, as confirmed by several researchers [10], [11]. Also shown is the "strong coupling" curve $T_{\mathrm{e}}=T_{\mathrm{ph}}$ (dashed line), which can be considered a "hard" lower bound.

It is seen that the fit temperatures indeed saturate at low MC temperatures, as suggested by the work by Wellstood et al. [16]. However, attempts to make the results fit to the $T_{\mathrm{e}}=\sqrt[5]{T_{0}^{5}+T_{\mathrm{ph}}^{5}}$ form, which applies for uniform heating in a thin film, seem to fail ( $T_{0}$ is the temperature at which $T_{\mathrm{e}}$ saturates for $T_{\mathrm{ph}} \rightarrow 0$ ). A much more reasonable fit is provided by the form $T_{\mathrm{e}}=\sqrt[2.5]{T_{0}^{2.5}+T_{\mathrm{ph}}^{2.5}}$ (solid line). This form respects the lower bound as well as the limit at $T_{\mathrm{ph}} \rightarrow 0$. Such a dependence is not entirely unreasonable, considering that the heating is localized in the SET transistor, and that heat transfer is provided only by the leads, which should probably be considered between one- and two-dimensional. Indeed the experiments of Wellstood et al. on local heating of a SQUID with cooling fins gave a $T_{\mathrm{e}}^{2.7}$ form.

We will adopt the $T_{\mathrm{e}}=\sqrt[2.5]{T_{0}^{2.5}+T_{\mathrm{ph}}^{2.5}}$ form as our estimate of the temperature in the following. We make no claims that this is the accurate temperature, only that it seems to be a reasonable approximation which makes a sensible transition from the lowtemperature to the high-temperature regime.

\section{C. $V_{\mathrm{RF}}^{2}$ Dependence}

In this section, we present measurements at maximum blockade, which means that $\Delta^{ \pm}$reduce to the charging energy $E_{\mathrm{C}}$. Also, since there is no asymmetry in the device at zero bias

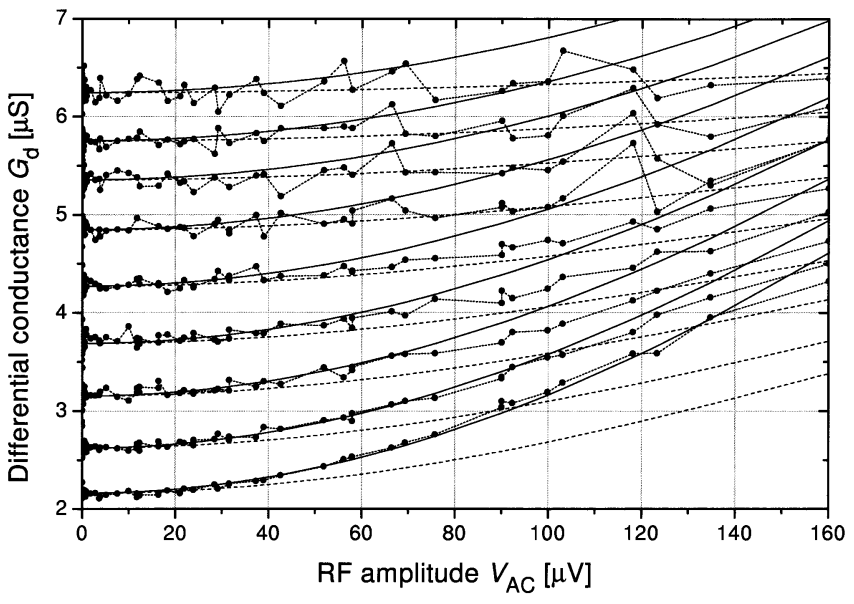

Fig. 3. Differential conductance for 9 different temperatures and for all frequencies marked in Fig. 1 as function of the RF amplitude at $37.9 \mathrm{~dB}$ additional damping. The measured values (points) are connected to guide the eye. The lines are the measured zero-bias value plus the predicted excess conductance with cotunneling (solid) and without cotunneling (dashed). The predictions are at 232, 255, 275, 301, 336, 379, 428, 478, and $549 \mathrm{mK}$, respectively (bottom to top).

and maximum blockade (no tunneling direction is favored), there should be no frequency dependence in the differential conductance. This enables us to find the relative damping of the line at the individual frequencies. Without frequency dependence, the zero bias conductance $G_{\mathrm{D}}$ as function of the microwave amplitude $V_{\mathrm{RF}}$ should be the same at all frequencies. We use subscript RF to distinguish from the $2 \mathrm{~Hz}$ modulation, used for measurement of $G_{\mathrm{D}}$. This means that the relative damping can be found by minimizing the mean square difference between the $G_{\mathrm{D}}-V_{\mathrm{RF}}$ curves at the individual frequencies, using the damping as free parameter. This procedure provided us with the relative damping values $0.0,3.8,9.1$, $11.5,14.817 .3$, and $17.6 \mathrm{~dB}$ for the frequencies marked in Fig. 1. As expected from Fig. 1, the damping increases with frequency. However, it should be noted that the optimal values vary slightly with temperature, suggesting that there might be a co-dependence on temperature and frequency.

The additional damping (i.e., the damping that should be added to the relative damping to get the absolute damping) should be the same at all frequencies and temperatures. Unfortunately, the absolute damping is unknown. All we can do is to find a damping value that is consistent with theory. In Fig. 3, the resulting theoretical prediction of the $G_{D}-V_{R F}$ curves at nine different temperatures are shown along with the measured values for all frequencies marked in Fig. 1, assuming an additional damping of $37.9 \mathrm{~dB}$. It is seen that the curves from different frequencies have indeed collapsed into one, especially at the lowest temperature. At higher temperatures, where the change in tunneling is more modest, the picture is not as perfect. Also shown is the predicted excess conductance with and without cotunneling at the same temperature added to the zero-power conductance. It is seen that the measured conductance fits the predicted one quite well, especially at low temperatures.

One "sanity check" that should be performed is whether the orthodox theory alone can explain the results. Using the value 


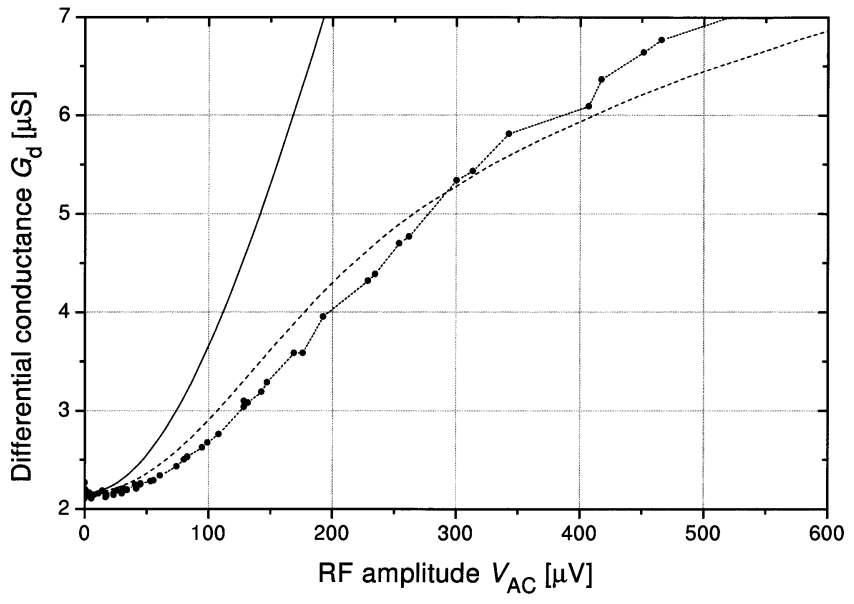

Fig. 4. Differential conductance as function of RF amplitude at $36.2 \mathrm{~dB}$ additional damping. The data (points) are the same as the bottom curves in Fig. 3, except that a lower damping in the microwave line has been assumed. The lines are the measured zero-bias value plus the predicted excess conductance with cotunneling (solid) and without cotunneling (dashed), for a temperature of $257 \mathrm{mK}$. This is the electron temperature fitted from the maximum blockade conductance (compare the squares in Fig. 2). Note that the horizontal scale is different from that in Fig. 3.

$36.2 \mathrm{~dB}$ for the additional damping gives the result in Fig. 4. It is seen that the results cross the prediction for the orthodox tunneling at this damping value. At higher damping, the conductance would be too high at high amplitudes, while a lower damping the conductance would be too low at low amplitudes. Thus the results can not be explained by the orthodox theory, even if one assumes a higher electron temperature and another damping. The electron temperature would also have to be substantially larger than at the MC at all temperatures, as seen in Fig. 2. Note that it would seem that we are applying a double standard here, since the horizontal scale is different in Figs. 3 and 4 . However, the orthodox theory should be valid for all voltages and thus amplitudes, while the cotunneling theory only claims validity for $e V_{\mathrm{RF}} \ll \Delta^{ \pm}$.

\section{Discussion AND CONCLUSION}

The enhancement of cotunneling by a coherent microwave source has been investigated experimentally and compared with theory. The results on cotunneling seem to confirm the theory for adiabatic enhancement of cotunneling by a coherent microwave signal. However, the presence of assumptions and adjustable parameters should inspire modesty in the strength of the conclusions drawn. Furthermore, the results seem to suggest that the effect of microwave amplitude and temperature are not independent, contrary to what is suggested by theory. Thus a more complete theory is needed. In any case, the presence of microwave induced cotunneling enhances the importance of taking cotunneling into account in the calculation of SET devices.

The interpretation of the results involved several explicit and implicit assumptions. To determine the lowest temperatures, it was assumed that both the orthodox theory for sequential tunneling and the original cotunneling theory by Averin and Nazarov [8] were correct within the given bounds of validity. The orthodox theory has been extensively confirmed since the first theories were published, and also the original cotunneling has been verified [10], [11]. It was further assumed that the coupling from the electron system to the MC through the phonon system was strong enough at high temperatures to assume a convergence toward the MC temperature here.

Future measurements should focus on verifying the photonassisted current in (3), e.g., by biasing in zero voltage and observing the current as function of amplitude and frequency. A careful determination of the device parameters and damping of the microwave line is essential, as there will be adiabatic contributions from both sequential tunneling and cotunneling. Also the measurements should be performed in several samples to rule out sample-specific effects.

\section{ACKNOWLEDGMENT}

The authors would like to thank K. Flensberg, M. Ejrnæs, M. R. Samuelsen, and the SET group at PTB, Braunschweig, Germany for collaboration and fruitful discussions.

\section{REFERENCES}

[1] R. L. Kautz, M. W. Keller, and J. M. Martinis, "Leakage and counting errors in a seven-junction electron pump," Phys. Rev. B, vol. 60, pp. 8199-8212, Sept. 1999.

[2] M. W. Keller, A. L. Eichenberger, J. M. Martinis, and N. M. Zimmerman, "A capacitance standard based on counting electrons," Science, vol. 285, pp. 1706-1709, Sept. 1999.

[3] A. Aassime, D. Gunnarsson, K. Bladh, P. Delsing, and R. J. Schoelkopf, "Radio-frequency single-electron transistor: Toward the shot-noise limit," Applied Physics Letters, vol. 79, pp. 4031-4033, Dec. 2001.

[4] J. P. Kauppinen, K. T. Loberg, A. J. Manninen, and J. P. Pekola, "Coulomb blockade thermometer: Tests and instrumentation," Rev. Sci. Instrum., vol. 69, pp. 4166-4175, Dec. 1998.

[5] T. Bergsten, T. Claeson, and P. Delsing, "A fast, primary coulomb blockade thermometer," Appl. Phys. Lett., vol. 78, pp. 1264-1266, Feb. 2001.

[6] D. V. Averin and K. K. Likharev, "Single-electronics: Correlated transfer of single electrons and cooper pairs in small tunnel junctions," in Mesoscopic Phenomena in Solids, B. L. Altshuler, P. A. Lee, and R. A. Webb, Eds: Elsevier, 1991, vol. 30, Modern Problems in Condensed Matter Physics, ch. 6, pp. 173-271.

[7] G.-L. Ingold and Y. V. Nazarov, "Charge tunneling rates in ultrasmall junctions," in Single Charge Tunneling, H. Grabert and M. H. Devoret, Eds: Plenum, 1992, pp. 21-108.

[8] D. V. Averin and Y. V. Nazarov, "Virtual electron diffusion during quantum tunneling of the electric charge," Phys. Rev. Lett., vol. 65, no. 19, pp. 2446-2449, 1990.

[9] D. V. Averin and A. A. Odintsov, "Macroscopic quantum tunneling of the electric charge in small junctions," Physics Letters A, vol. 140, pp. 251-257, Sept. 1989

[10] L. J. Geerlings, D. V. Averin, and J. E. Mooij, "Observation of macroscopic quantum tunneling through the coulomb energy barrier," Physical Review Letters, vol. 65, pp. 3037-3040, Dec. 1990.

[11] T. M. Eiles, G. Zimmerli, H. D. Jensen, and J. M. Martinis, "Thermal enhancement of cotunneling in ultra-small tunnel junctions," Phys. Rev. Lett., vol. 69, pp. 148-151, July 1992.

[12] C. Pasquier, U. Meirav, F. I. B. Williams, and D. C. Glattli, "Quantum limitation on coulomb blockade observed in a 2 d electron system," Physical Review Letters, vol. 70, pp. 69-72, Jan. 1993.

[13] M. Covington, M. W. Keller, R. L. Kautz, and J. M. Martinis, "Photonassisted tunneling in electron pumps," Phys. Rev. Lett., vol. 84, pp. 5192-5195, May 2000.

[14] K. Flensberg, "Coherent-photon-assisted cotunneling in a coulomb blockade device," Phys. Rev. B, vol. 55, pp. 13 118-13 123, May 1997.

[15] M. Manscher, M. Savolainen, and J. Mygind, "Experimental investigation of microwave enhanced cotunneling in set transistors," Phys. Rev. B, Jul. 26, 2002. July arXiv:cond-mat/0 207639 vl, submitted for publication.

[16] F. C. Wellstood, C. Urbina, and J. Clarke, "Hot-electron effects in metals," Phys. Rev. B, vol. 49, pp. 5942-5955, Mar. 1994. 